\title{
INTERESTERIFICAÇÃO ENZIMÁTICA CONTÍNUA DA GORDURA DO LEITE COM ÓLEO DE SOJA EM REATOR DE LEITO FLUIDIZADO: AVALIAÇÃO DA CONSISTÊNCIA E DO CONTEÚDO DE GORDURA SÓLIDA
}

\author{
A. V. de Paula ${ }^{1}$; G. F. M. Nunes ${ }^{2}$; H. F. de Castro ${ }^{2}$; S. Ferreira-Dias ${ }^{3}$; J. C. dos Santos ${ }^{4}$ \\ ${ }^{1}$ Univ Estadual Paulista, UNESP, Departamento de Bioprocessos e Biotecnologia \\ ${ }^{2}$ Univ de São Paulo, USP, Departamento de Engenharia Química \\ ${ }^{3}$ Univ de Lisboa, Instituto Superior de Agronomia \\ ${ }^{4}$ Univ de São Paulo, USP, Departamento de Biotecnologia \\ E-mail para contato: ariela@fcfar.unesp.br; jsant200@usp.br
}

\begin{abstract}
RESUMO - Reatores de leito fluidizado apresentam vantagens como boas condições de mistura do meio sem uso de agitadores mecânicos que poderiam danificar biocatalisadores imobilizados. Neste trabalho, esta configuração de reator foi utilizada para a interesterificação de uma mistura composta por $65 \%$ de gordura de leite e $35 \%$ de óleo de soja catalisada por preparação comercial de lipase de Candida antarctica imobilizada (Novozym ${ }^{\circledR 435) . ~ O ~ t e m p o ~ e s p a c i a l ~ f o i ~ d e ~} 37$ minutos e a reação contínua foi mantida por $168 \mathrm{~h} \mathrm{a} 45^{\circ} \mathrm{C}$, monitorando-se a consistência e o conteúdo de gordura sólida (CGS), obtido por espectroscopia de ressonância magnética nuclear. Obteve-se uma porcentagem de redução de $34 \%$ na consistência dos produtos interesterificados em relação à mistura inicial e, com relação ao $\mathrm{CGS}$, os resultados obtidos $\left(\mathrm{CGS} 10^{\circ} \mathrm{C}=28,18 ; \mathrm{CGS} 20^{\circ} \mathrm{C}=12,57\right.$; $\mathrm{CGS} 35^{\circ} \mathrm{C}=0,29$ ) apresentaram-se dentro das faixas consideradas ideais para "spreads" com satisfatória espalhabilidade.
\end{abstract}

\section{INTRODUÇÃO}

Entre os alimentos apreciados pelo consumidor encontra-se a gordura de leite, à qual se atribui sabor e aroma peculiares e agradáveis, além de possuir características importantes e benéficas à saúde humana, como a presença de ácidos linoléicos conjugados (Firestone, 2006; Shin et al., 2009). No entanto, para a aceitabilidade de um produto é fundamental que se considerem aspectos como as propriedades físicas e sensoriais. Neste caso, a gordura de leite tem como desvantagem baixa espalhabilidade sob temperatura de refrigeração doméstica (Rodrigues $e t$ al., 2003). Nesse sentido, a substituição da manteiga por margarinas ou cremes vegetais tem levado a indústria a buscar processos que permitam modificar a gordura do leite, visando ao aumento das alternativas para uso desta matéria prima (Balcão et al., 1998).

Entre os processos considerados, a interesterificação química ou enzimática permite a alteração das propriedades de espalhabilidade, devido à modificação nas propriedades físicas da manteiga introduzida pela redistribuição dos ácidos graxos entre os triacilgliceróis (Rodrigues e Gioielli, 2003). No caso da interesterificação enzimática são empregadas lipases, enzimas hidrolíticas de grande aplicação industrial (Alim et al., 2008). Algumas limitações da via 
enzimática incluem o preço da enzima e a baixa estabilidade operacional das lipases (Osório et al., 2006), limitações estas que podem ser contornadas empregando-se técnicas de imobilização, permitindo assim o aumento da produtividade do processo (Zanin et al., 2004).

A escolha do tipo de reator a ser empregado em determinado processo está relacionada à forma e às características do derivado imobilizado, além de determinados requisitos operacionais. Quando suportes de imobilização possuem dimensões muito pequenas não podem ser empregados em reatores de leito fixo, uma vez que a perda de carga ao longo do reator é muito elevada e, particularmente quando se opera em fluxo descendente, pode ocorrer a compactação do leito (Fernandes et al., 2003). Vários dos problemas que ocorrem com leito fixo podem ser solucionados empregando-se reatores de leito fluidizado, que proporcionam um grau de mistura intermediário entre os reatores agitados e os de leito fixo. As velocidades de fluido que são atingidas permitem minimizar os efeitos de transferência de massa externos que podem se fazer presentes em reatores de leito fixo ou em casos em que se utiliza agitação suave (Fernandes et al., 2003; Teixeira et al., 2007). As vantagens dos reatores de leito fluidizado incluem a eliminação da formação de caminhos preferenciais, menor alteração da pressão quando altas vazões de alimentação são empregadas, e redução de gradientes de concentração (Willis e Marangoni, 2008).

Nesse contexto, o presente trabalho teve como objetivo avaliar o emprego de reator de leito fluidizado para modificação da gordura do leite por interesterificação com óleo de soja, empregando-se preparação comercial de lipase de Candida antarctica imobilizada (Novozym ${ }^{\circledR 435) . ~ O ~ a c o m p a n h a m e n t o ~ d a ~ r e a c ̧ a ̃ o ~ f o i ~ e f e t u a d o ~ a t r a v e ́ s ~ d a ~ q u a n t i f i c a c ̧ a ̃ o ~ d a ~}$ consistência e do conteúdo de gordura sólida (CGS).

\section{MATERIAIS E MÉTODOS}

\subsection{Materiais}

Lipase: o experimento foi efetuado com a lipase comercial Novozym ${ }^{\circledR} 435$ (Novozymes A/S, Bagsvaerd, Denmark), de Candida antarctica.

Matérias primas: gordura de leite obtida a partir de fusão completa de manteiga comercial (Mimosa, sem sal) sob temperatura de $50-65^{\circ} \mathrm{C}$ em forno de micro-ondas para desfazer a emulsão, seguida de centrifugação e separação da fase aquosa; óleo de soja comercial (Óleo de soja refinado, Sovena Portugal Consumer Goods, S. A), refinado sem tratamento adicional.

\subsection{Metodologia}

Interesterificação em leito fluidizado: o reator de leito fluidizado (altura de $20 \mathrm{~cm}$, diâmetro interno de 2,0 cm) foi operado em modo contínuo por 7 dias. O meio reacional foi composto de $65 \%(\mathrm{~m} / \mathrm{m})$ de gordura de leite, $35 \%(\mathrm{~m} / \mathrm{m})$ de óleo de soja e a temperatura empregada foi $45^{\circ} \mathrm{C}$. A reação foi catalisada por Novozym ${ }^{\circledR} 435(7,6 \mathrm{~g})$, com tempo espacial de 37 min e vazão de alimentação de $0,76 \mathrm{~mL} / \mathrm{min}$ (Paula, 2012). Um esquema do sistema utilizado é apresentado na Figura 1. 


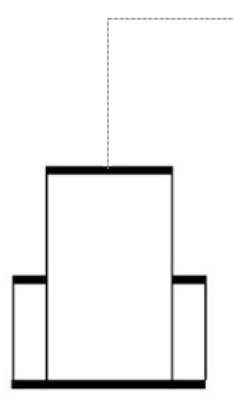

T1

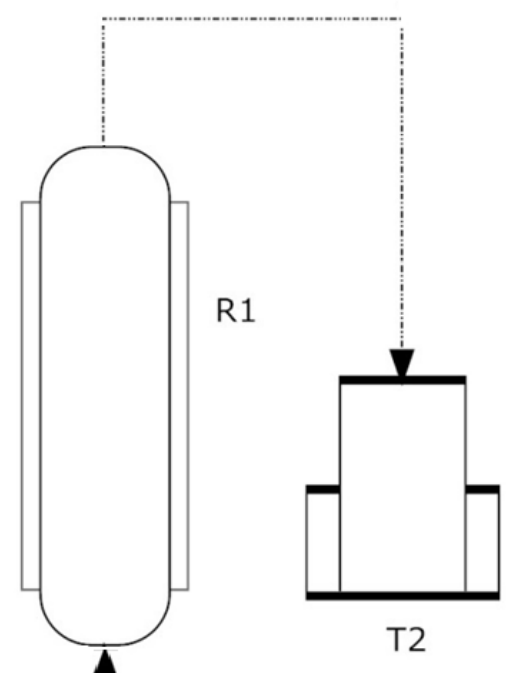

Figura 1 - Fluxograma simplificado do reator de leito fluidizado empregado nas reações de interesterificação da gordura de leite com óleo de soja: sistema contínuo. T1.

Reservatório de alimentação; T2. Reservatório de produtos; R1. Reator de Coluna; P1.

Bomba peristáltica. ( (........) Linha de alimentação $;(-\cdots-\cdots-)$ Linha de produtos.

A reação de interesterificação da gordura de leite e óleo de soja, catalisada por Novozym ${ }^{\circledR}$ 435 com densidade cristalina de 0,833 $\pm 0,067$ (valor médio \pm desvio padrão), foi efetuada empregando-se 7,6 g de derivado imobilizado e uma vazão de $0,76 \mathrm{~mL} / \mathrm{min}$, a qual foi mantida por 7 dias. Após a fluidização, a altura do leito passou a ser $12 \mathrm{~cm}$, correspondendo a um volume útil de $28,55 \mathrm{~mL}$ e tempo espacial de 37 minutos.

O progresso da reação foi acompanhado pela determinação do conteúdo de gordura sólida (CGS) e da consistência dos produtos interesterificados.

Conteúdo de gordura sólida por espectroscopia de ressonância magnética nuclear (RMN): as análises de conteúdo de gordura sólida foram efetuadas por espectroscopia de ressonância magnética nuclear (RMN), de acordo com a metodologia descrita por Osório et al. (2009).

Consistência: a consistência das amostras foi determinada utilizando texturômetro (QTS-25 Brookfield). As amostras foram aquecidas em forno microondas $\left(45-50^{\circ} \mathrm{C}\right)$, para fusão completa dos cristais, e condicionadas em béquer de vidro $(100 \mathrm{~mL})$. O condicionamento foi efetuado por $48 \mathrm{~h}$ em estufa a temperatura controlada $\left(10^{\circ} \mathrm{C}\right)$ para recristalização da gordura. Foi utilizada a sonda TA15, correspondente a um cone acrílico com ponta não truncada e ângulo de $45^{\circ}$. Os testes foram conduzidos nas seguintes condições: retorno ao início, distância: $10 \mathrm{~mm}$, velocidade: $120 \mathrm{~mm} / \mathrm{min}$, tempo: $5 \mathrm{~s}$, determinação da força em compressão (gf), em duplicata. As amostras foram analisadas quanto ao "yield value" que foi calculado pela Equação 1, proposta por Haighton (1959): 


$$
\mathrm{C}=\mathrm{K} \cdot \frac{\mathrm{W}}{\mathrm{p}^{1.6}}
$$

em que: $\mathrm{C}=$ consistência ("yield value"; $\mathrm{gf} / \mathrm{cm}^{2}$ ), $\mathrm{K}=$ fator dependente do ângulo do cone (para $\left.45^{\circ}, 4700\right), \mathrm{W}=$ força máxima em compressão (gf), para tempo de $5 \mathrm{~s}, \mathrm{p}=$ profundidade de penetração $(0,1 \mathrm{~mm})$.

\section{RESULTADOS E DISCUSSÃO}

\subsection{Consistência}

A consistência é um aspecto funcional importante de gorduras plásticas, constituídas de cristais de gordura sólida e óleo líquido, sendo um fator crítico para determinação da funcionalidade e aceitação do consumidor por gorduras interesterificadas (Silva et al., 2009). De acordo com Haighton (1959), para que uma gordura interesterificada apresente satisfatórias propriedades de plasticidade e espalhabilidade, deve apresentar valores de consistência na faixa de 200-800 gf $/ \mathrm{cm}^{2}$, sendo esta adotada como parâmetro referencial para este trabalho. Os resultados de consistência dos produtos interesterificados (valor médio \pm desvio padrão) são apresentados na Figura 2.

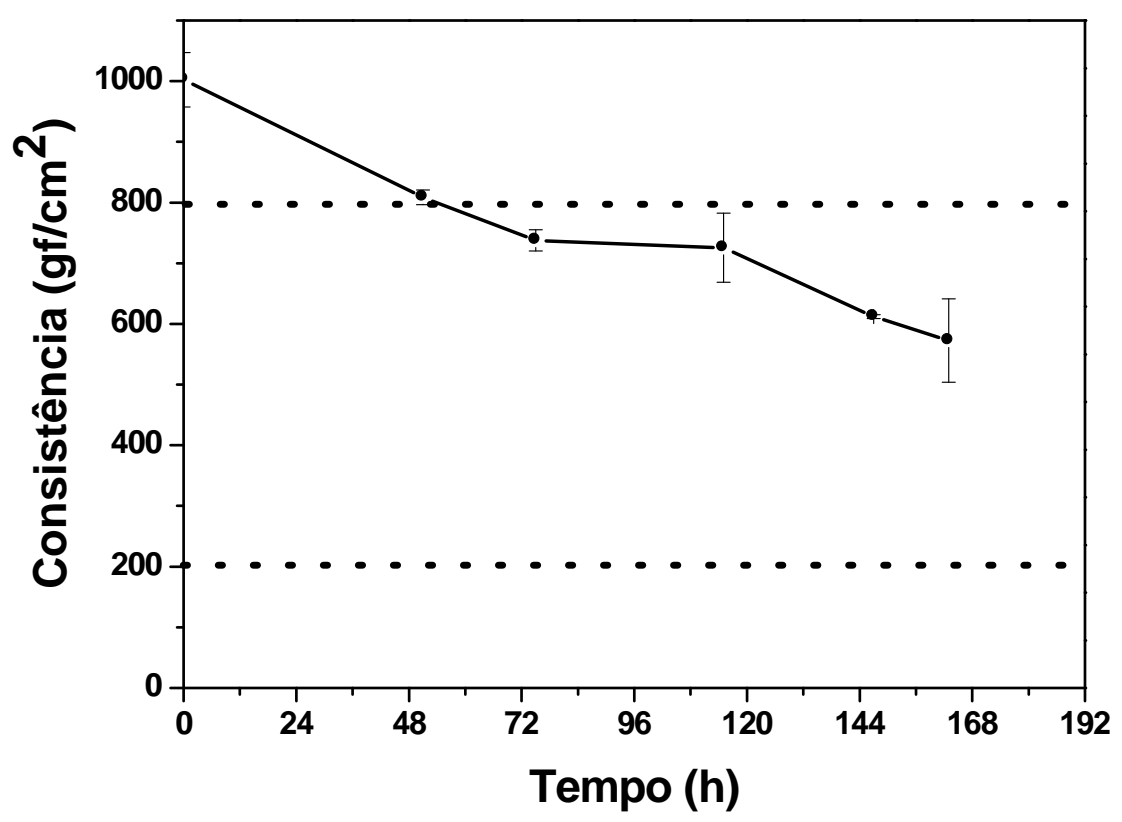

Figura 2 - Consistência $\left(\mathrm{gf} / \mathrm{cm}^{2}, \bullet\right)$ dos produtos interesterificados obtidos na reação de interesterificação da gordura de leite e óleo de soja (mistura 65\%:35\%) catalisada por Novozym ${ }^{\circledR}$ 435 , em reator de leito fluidizado

Os resultados da Figura 2 revelam que todos os produtos interesterificados, a partir de $75 \mathrm{~h}$ 
de operação, atingiram valores de consistência dentro da faixa ideal (200-800 gf/ $\left.\mathrm{cm}^{2}\right)$, mantendose praticamente constante até o final. Os valores de redução percentual das consistências dos produtos interesterificados, em relação à consistência $\left(1002,43 \pm 44,65 \mathrm{gf} / \mathrm{cm}^{2}\right)$ da mistura inicial (65:35 gordura de leite:óleo de soja) são apresentados na Tabela 1.

Tabela 1. Redução percentual da consistência dos produtos interesterificados em relação à mistura reacional inicial, obtido na reação de interesterificação da gordura de leite e óleo de soja catalisada por Novozym ${ }^{\circledR} 435$, em reator de leito fluidizado, empregando-se tempo espacial de 37 $\min$

\begin{tabular}{cc}
\hline Tempo (h) & \% Redução \\
\hline $\mathbf{5 1}$ & 19,34 \\
\hline $\mathbf{7 5}$ & 26,42 \\
\hline $\mathbf{1 1 5}$ & 27,64 \\
\hline $\mathbf{1 4 7}$ & 38,94 \\
\hline $\mathbf{1 6 3}$ & 42,88 \\
\hline *65\%:35\% gordura de leite:óleo de soja
\end{tabular}

Calculando-se a média dos valores da Tabela 1 no estado estacionário (a partir de $75 \mathrm{~h}$ ), obteve-se consistência de $661,89 \pm 82,11 \mathrm{gf} / \mathrm{cm}^{2}$, o que representa porcentagem de redução média de $33,97 \pm 8,19 \%$, em relação à consistência da mistura inicial, sendo este um resultado bastante satisfatório.

\subsection{Conteúdo de Gordura Sólida (CGS)}

O conteúdo de gordura sólida (CGS) indica o percentual de gordura que se encontra no estado sólido a uma determinada temperatura (Gunstone et al., 2007). Esta propriedade é responsável por muitos atributos importantes das gorduras, por exemplo, aparência física, características sensoriais, espalhabilidade, características de fusão, e plasticidade ou consistência de um produto alimentício.

O conteúdo de gordura sólida entre 4 e $10^{\circ} \mathrm{C}$ determina a espalhabilidade do produto na temperatura de refrigeração. CGS não superior a $32 \%$ à temperatura de $10^{\circ} \mathrm{C}$, é indicado para garantir boa espalhabilidade nesta temperatura. O CGS do produto entre 20 e $22^{\circ} \mathrm{C}$ determina sua estabilidade e resistência à exsudação do óleo. Neste caso, o teor ideal não deve ser inferior a $10 \%$ (O'Brien, 2009; Ribeiro et al., 2009). O conteúdo CGS entre 33 e $38^{\circ} \mathrm{C}$ influencia as impressões e sensações de arenosidade do alimento na boca (O’Brien, 2009; Otero et al., 2006).

A determinação do conteúdo de gordura sólida foi efetuada por RMN, em três diferentes temperaturas: 10,20 e $35^{\circ} \mathrm{C}\left(\mathrm{CGS}_{10^{\circ} \mathrm{C}}, \mathrm{CGS}_{20^{\circ} \mathrm{C}}\right.$ e $\left.\mathrm{CGS}_{35^{\circ} \mathrm{C}}\right)$, e os resultados são apresentados na Figura 3. 


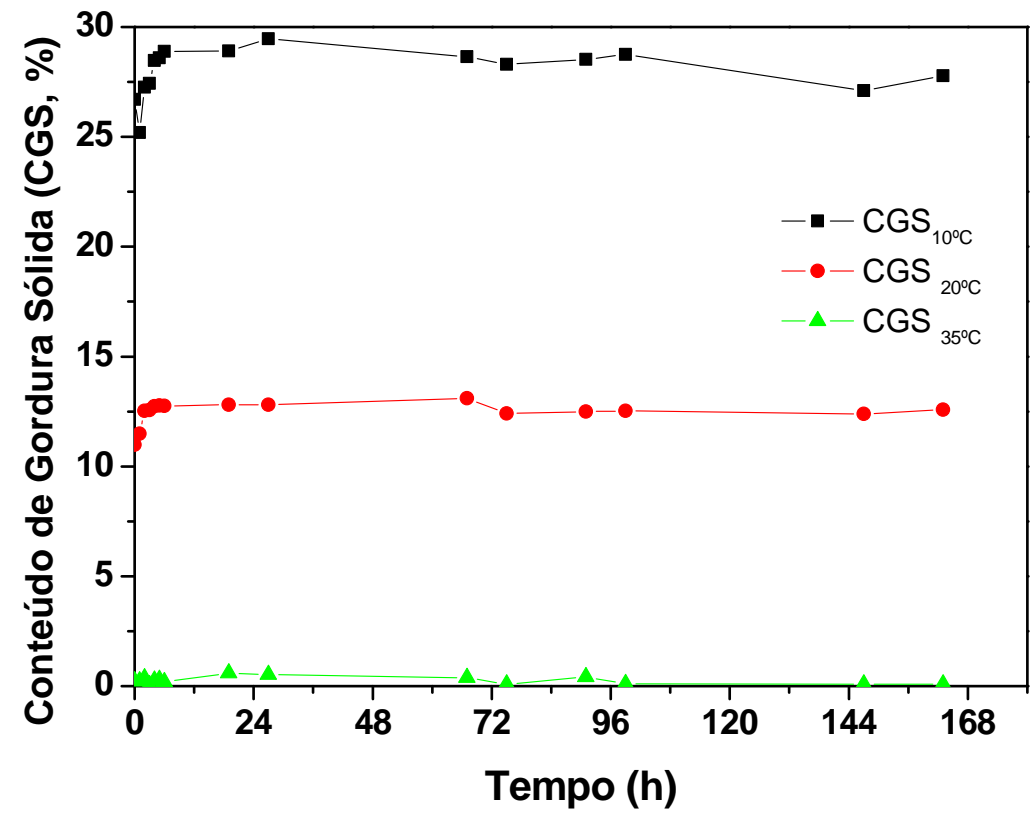

Figura 3. Conteúdo de Gordura Sólida (CGS), em diferentes temperaturas, quantificados ao longo da reação de interesterificação da gordura de leite e óleo de soja (mistura 65\%:35\%) catalisada por Novozym ${ }^{\circledR} 435$, em reator de leito fluidizado

Os produtos interesterificados apresentaram os seguintes valores (valor médio \pm desvio padrão) de CGS, no estado estacionário: $\mathrm{CGS}_{10^{\circ} \mathrm{C}}=28,18 \pm 0,70 ; \mathrm{CGS}_{20^{\circ} \mathrm{C}}=12,57 \pm 0,37 \mathrm{e}$ $\mathrm{CGS}_{35^{\circ} \mathrm{C}}=0,29 \pm 0,17$. Com relação às misturas iniciais, os seguintes valores (valor médio \pm desvio padrão) de CGS eram: $\mathrm{CGS}_{10^{\circ} \mathrm{C}}=26,70 \pm 0,67$ CGS $_{20^{\circ} \mathrm{C}}=11,00 \pm 0,08$ e $\mathrm{CGS}_{35^{\circ} \mathrm{C}}=0,23 \pm$ 0,09. Os valores de CGS apresentam-se dentro das respectivas nas faixas ideais, em todas as temperaturas estudadas. Além disso, uma pequena elevação no CGS ocorreu nas primeiras horas de reação. Após este período, os valores de $\mathrm{CGS}_{10^{\circ} \mathrm{C}}$ e $\mathrm{CGS}_{20^{\circ} \mathrm{C}}$ mantiveram-se praticamente constantes.

Os resultados mostram que os valores de CGS médio em comparação ao CGS das misturas iniciais, nas respectivas temperaturas foram próximos, variando muito pouco. $\mathrm{Na}$ temperatura de $10^{\circ} \mathrm{C}$ observou-se um leve aumento no CGS em relação à mistura inicial. Resultados semelhantes foram obtidos por Zhang et al. (2006), que estudaram a interesterificação da gordura de leite com óleo de colza, catalisada por Lipozyme TL IM, a $70{ }^{\circ} \mathrm{C}$, objetivando demonstrar a aplicação da espectroscopia FTNIR para monitoramento da reação, em comparação ao método do CGS.

\section{CONCLUSÃO}

Os resultados revelaram que a Novozym ${ }^{\circledR} 435$ apresenta-se como um biocatalisador com potencial uso na para modificação da gordura de leite, uma vez que apresentou boa redução percentual da consistência $(33,97 \pm 8,19 \%)$ dos produtos interesterificados. O uso deste biocatalisador permitiu a obtenção de um produto interesterificado com satisfatórias propriedades 
de plasticidade e espalhabilidade, em reator de leito fluidizado operado em modo contínuo, sendo que os resultados obtidos podem servir de base a estudos visando-se à utilização de outras opções de biocatalisador e ampliação de escala.

\section{AGRADECIMENTOS}

Os autores agradecem à FAPESP, ao CNPq e à CAPES pelo suporte financeiro.

\section{NOMENCLATURA}

$\begin{array}{ll}\text { RMN } & \text { Ressonância Magnética Nuclear } \\ \text { CGS } & \text { Conteúdo de gordura sólida }\end{array}$

\section{REFERÊNCIAS}

ALIM, M. A.; LEE, J. H.; SHIN, J. A.; LEE, Y. J.; CHOI, M. S.; AKOH, C. C.; LEE, K. T. Lipase-catalyzed production of solid fat stock from fractionated rice bran oil, palm stearin, and conjugated linoleic acid by response surface methodology. Food Chem., v. 106, p. 712-719, 2008.

BALCÃO, V. M.; KEMPPINEM, A.; MALCATA, F. X.; KALO, P. J. Modification of butterfat by selective hydrolysis and interesterification by lipase: Process and product characterization. $J$. Am. Oil Chem. Soc., v. 7 5, p. 1347-1358, 1998.

FERNANDES, P; FERREIRA, B. S.; CABRAL, J. M. S. Reactores enzimáticos Multifásicos; Em: J. CABRAL, M.S.; AIRES-BARROS, M. R.; GAMA, M. Engenharia enzimática. Lisboa: Lidel, 2003. cap. 8, p. 189-208.

FIRESTONE, D. Physical and Chemical Characteristics of Oils, Fats, and Waxes. 2nd ed. Washington: AOCS PRESS, 2006. 237 p. ISBN: 978-1-893997-99-8.

GUNSTONE, F. D.; HARWOOD, J. L.; DIJKSTRA, A. J. The Lipid Handbook. 3rd.ed. Boca Raton: CRC Press, 2007. 791p.

HAIGHTON, A. J. The measurement of the hardness of margarine and fats with cone penetrometers. J. Am. Oil Chem. Soc., v. 36, n. 8, p. 345-348, 1959.

O'BRIEN, R. D. Fats and oils: Formulating and processing for applications. 2 ed. Florida: CRC Press, 2009.

OSÓRIO, N. M.; DUBREUCQ, E.; DA FONSECA, M. M. R.; FERREIRA-DIAS, S. Operational stability of immobilised lipase/ acyltransferase during interesterification of fat blends. Eur. J. Lipid Sci. Technol, v. 111, n. 4, p. 358-367, 2009.

OSÓRIO, N. M.; FONNSECA, M. M; FERREIRA-DIAS, S. Operational stability of Thermomyces lanuginosa lipase during interesterification of fat in continuous packed-bed reactors. Eur. J. Lipid Sci. Technol., v. 108, p. 545-553, 2006. 
OTERO, C.; LÓPEZ-HERNANDEZ, A.; GARCÍA, H. S.; HERNÁNDEZ-MARTÍN, E.; HILL JR., C. G. Continuous enzymatic transesterification of sesame oil and a fully hydrogenated fat: effects of reaction conditions on product characteristics. Biotechnol. Bioeng., v. 94, n. 5, 2006.

PAULA, A. V. Reestruturação da gordura de leite por interesterificação enzimática empregando lipase imobilizada: otimização das condições reacionais e operacionais. 2011. 212 p. Tese (Doutorado em Ciências) - Escola de Engenharia de Lorena, Universidade de São Paulo, Lorena/SP, 2012.

RIBEIRO, A.P. B.; BASSO, R. C.; GRIMALDI, R.; GIOIELLI, L. A.; GONÇALVES, L. A. G. Instrumental methods for the evaluation of interesterified fats. Food Anal. Methods, v. 2, p. 282302, 2009.

RODRIGUES, J. N.; GIOIELLI, L. A. Chemical interesterification of milkfat and milkfat-corn oil blends. Food Res. Int., v. 36, p. 149-159, 2003.

RODRIGUES, J. N.; GIOIELLI, L. A.; ANTON, C. Propriedades físicas de lipídios estruturados obtidos de misturas de gordura do leite e óleo de milho. Ciência Tecnol. Alimentos, v. 23, p. 226233, 2003.

SHIN, J. A.; AKOH, C. C.; LEE, K. T. Production and physicochemical properties of functional - butterfat through enzymatic interesterification in a continuous reactor. J. Agri. Food Chem., v. 57, n. 3, p. 888-900, 2009.

Silva, R. C.; COTTING, L. N.; POLTRONIERI, T. P.; BALCAO, V. M.; DE ALMEIDA, D. B.; GONCALVES, L. A. G.; GRIMALDI, R.; GIOIELLI, L. A. The effects of enzymatic interesterification on the physical - chemical properties of blends of lard and soybean oil. LWT-Food Sci. Tech., v. 42, n. 7, p. 1275-1282, 2009.

TEIXEIRA, J. A.; FONSECA, M. M.; VICENTE, A. Geometria e modos de operação. Em: FONSECA, M. M.; TEIXEIRA, J. A. Reactores Biológicos: Fundamentos e Aplicações. Lisboa: Lidel, 2007.

WILLIS, W. M.; MARANGONI, A. G. Enzymatic Interesterification. In: AKOH, C. C. and MIN, D. B. Food lipids: chemistry, nutrition, and biotechnology. Florida: CRC Press, 2008. Cap. 30. p. 807. ISBN 978-1-4200-4663-2.

ZANIN, G. M.; MORAES, F, F. Enzimas Imobilizadas. In SAID, S. e PIETRO, R. C. L. R. (ed.) Enzimas como agentes Biotecnológicos. Ribeirão Preto: Legis Summa, 2004. Cap. 4. p.35-85.

ZHANG, H., MU, H.; XU, X. Monitoring lipase-catalyzed butterfat interesterification with rapeseed oil by Fourier transform near-infrared spectroscopy. Anal. and Bioanal. Chem., v. 386, n. 6, p. 1889-1897, 2006. 